\title{
Retrospective chart review of catatonia in child and adolescent psychiatric patients
}

Ghaziuddin N, Dhossche D, Marcotte K. Retrospective chart review of catatonia in child and adolescent psychiatric patients.

Objective: Identify the frequency of catatonia among at-risk children and adolescents receiving psychiatric treatment.

Method: Subjects were children and adolescents ( $<18$ years), who had received psychiatric treatment at a University Hospital during 20042009, and were diagnosed with disorders with known risk for catatonia or displayed symptoms suggestive of catatonia. Approval was obtained from the Investigational Review Board (IRB). The first $101(n=101)$ subjects were selected among 570 subjects identified by psychiatric diagnoses: any pervasive developmental disorder, psychosis-NOS (Not Otherwise Specified), intermittent explosive disorder, mental retardation, catatonia and neuroleptic malignant syndrome. Subjects met study-defined criteria for catatonia, if they had three or more of the following symptoms: unexplained agitation/excitement, disturbed or unusual movements, reduced movements, repetitive or stereotyped movements, or reduced or loss of speech.

Results: Eighteen (17.8\%) subjects, among a group suspected to be at a higher risk for catatonia, met the study-defined criteria for this syndrome. However, only two subjects had been diagnosed by their treatment providers. Higher rates of intellectual disability and aggression were found among the group that met study-criteria.

Conclusion: We concluded that catatonia is under recognized and undertreated among children and adolescents receiving psychiatric treatment.

\section{N. Ghaziuddin ${ }^{1}$, D. Dhossche ${ }^{2}$, K. Marcotte ${ }^{3}$}

${ }^{1}$ Department of Psychiatry, University of Michigan, Ann Arbor, MI, USA, ${ }^{2}$ Department of Psychiatry, University of Mississippi Medical Center, Jackson, MS, USA and ${ }^{3}$ School of Nursing, University of Michigan, Ann Arbor, $\mathrm{MI}$, USA

Key words: child and adolescent psychiatry; catatonia Neera Ghaziuddin, University of Michigan, Ann Arbor, 4250 Plymouth Rd., Ann Arbor, MI 48109, USA.

E-mail: neerag@umich.edu

Accepted for publication September 20, 2011

\section{Significant outcomes}

- Catatonia is frequently missed among children and adolescents receiving psychiatric treatment. This appears to be secondary to lack of familiarity with the condition among clinicians.

- Patients with catatonia are not receiving treatments known to be effective for this condition (benzodiazepines, ECT (Electroconvulsive Therapy), and discontinuing antipsychotics).

- Agitated forms of catatonia, accompanied by aggression, are a common presentation among a subgroup of psychiatric patients who are at a higher risk for this syndrome.

\section{Limitations}

- Retrospective study design

- Variable data quality because of reliance on computer-assisted methodology.

\section{Introduction}

Catatonia is a syndrome characterized by motor abnormalities that include excessive movements (constant or periodic over-activity/excitement, unprovoked aggression or auto-aggression), reduced mobility (progressive slowness that may interfere with functioning, cessation of all movements in some cases), abnormal movements (repetitive stereotyped movements, mimicking the movements of others, grimacing, mannerisms, posturing, freezing), reduced or other abnormalities of 


\section{Ghaziuddin et al.}

speech (perseveration or senseless repetition of words or phrases, mimicking the speech of others, loss of ability to use sign language among non-verbal patients, or mutism among severe cases), reduced functioning (loss of skills such as toileting, ability to feed or dress), negativism (often senseless refusal to comply), and reduced food or fluid intake (may result in dangerous, medically compromised states). Symptoms may be present in different combinations, depending on illness-severity and premorbid functioning of the individual. The condition may persist for years, if not recognized and treated with effective treatments (1). In some severe cases, the condition may be accompanied by physical symptoms (cardiovascular in neuroleptic malignant syndrome; gastro-intestinal in serotonin syndrome) that are regarded by some as diverse manifestations of the same syndrome (2). It should be noted that the current classification system for psychiatric disorders, used in DSM-IV (diagnostic and statistical manual of mental disorders), only permits catatonia associated with medical conditions, or affective disorders and schizophrenia. Many experts in the field argue that catatonia should be acknowledged as a broad code in DSM5, and a non-coded catatonia specifier (proposed in revisions) will badly serve clinical practice and research $(3,4)$.

On a historical note, catatonia was originally described in 1874 by Kahlbaum (5) as a separate brain disorder with a cyclic, alternating, and a progressive course. Kahlbaum described the condition among a group of $(n=26)$ adult patients, ranging in age from 20 to 44 years. It was incorporated as a type of dementia praecox or schizophrenia by Kraepelin in 1896 (6). Since the 1970s, various authors have described catatonia to be a feature in affective disorders in adults, particularly mania (7-12).

Systematic studies of catatonia find that it is a relatively common condition among psychiatric patients; one cross-cultural study found rates ranging between $10 \%$ and $13 \%$ (13). Others have found that certain diagnostic groups may be at a relatively higher risk such as autism/pervasive developmental disorders (PDD) (14), mental retardation and other developmental disabilities (14), affective disorders $(15,16)$, and schizophrenia (17, 18). Among a long list of associated medical conditions are some specific conditions as encephalitis (19), meningitis (20), secondary to antipsychotic medications (21), toxins and infections (22-24). However, a high rate of $17 \%$ is noteworthy among patients with autism spectrum disorder/PDD $(14,25)$. Further, it has been suggested that there appear to be several areas of overlap between autism and catatonia (26).
Aims of the study

The present study was undertaken to identify the frequency of catatonia among children and adolescents at a mid-western university hospital, based on a retrospective chart review. Subjects were patients who had received psychiatric treatment, either during hospitalization or in outpatient clinics, were under the age of 18 years, were at an elevated risk for catatonia because they belonged to a risk group diagnosed with autism (alone or with mental retardation), were diagnosed with a disorder with a likely symptom overlap with catatonia (psychosis-NOS (Not Otherwise Specified), intermittent explosive disorder, mental retardation), and/or were already diagnosed with catatonia or neuroleptic malignant syndrome.

\section{Material and methods}

Data were initially collected for a quality improvement project and to increase awareness regarding catatonia among clinicians. Five hundred and seventy $(n=570)$ patients, under the age of 18 years, were identified from a University billing data base who had received psychiatric treatment between 2004 and 2009. Patients were selected if they had been diagnosed with any of the following psychiatric disorders: any pervasive developmental disorder (autistic disorder, PDD-NOS, Asperger syndrome), psychosis-NOS, intermittent explosive disorder, mental retardation, catatonia, or neuroleptic malignant syndrome (NMS). Investigational Review Board (IRB) approval was obtained for the publication of these data.

Next, among the subjects identified, hospital electronic medical records were searched, using EMERSE (Electronic Medical Record Search Engine), which is an electronic data search program and is used to identify symptoms of interest (whether present or absent) using key search terms (27). The key search terms used were as follows: aggression, unexplained excitement/agitation, disturbed or unusual movements, reduced movements, stereotyped or repetitive movements and behaviors, reduced or loss of speech, reduced appetite and reduced food or fluid intake. Each key term was entered in a variety of ways to identify all related symptoms, while diagnostic labels stated above were not re-entered at this point.

Further detailed data were collected for the first 101 subjects among this subgroup. These additional data could be only collected for a subgroup because of resource limitations. Data were demographic information, psychiatric and medical diagnoses, symptom details (symptom present/absent, 
severity and duration of symptom), psychotropic medications received, laboratory tests and outcome (if available).

Finally, the frequency of catatonia diagnosed by investigators was compared with the frequency by treating psychiatrists. For the purpose of this study, catatonia was defined as the presence of three or more of the following symptoms: unexplained excitement/agitation, unusual movements, reduced movements, repetitive or stereotyped movements, and reduced or loss of speech.

\section{Results}

The overall group of 101 subjects was predominantly male $=80.2 \%$, current age $=13.9 \pm 2.04$ years, age of first psychiatric contact in years $=$ $6.3 \pm 3.4$ (range $=1-14$ ); pervasive developmental disorder/autism spectrum disorder diagnosis $=81 \%$; the frequency of key symptoms in the overall group was excitement/agitation present $=75.2 \%$, disturbed/unusual movements $=$ $33 \%$, reduced speech $=29 \%$; and reduced mobility $/$ movement $=15 \%$. Reduced appetite $/$ reduced food intake was found in $=32 \%$ and reduced fluid intake in $=13 \%$. Creatinine phosphokinase enzyme (CPK) was checked in $5 \%$, but was recorded in medical documentation for only two subjects (level $=339$ and $677 \mathrm{IU} / 1$, respectively), and benzodiazepines were administered to $29 \%$.

Using our stringently defined criteria for catatonia (presence of three or more symptoms), 17.8\% subjects met investigator-based criteria for catatonia. Catatonia was diagnosed in only two subjects by the treatment providers. A variety of diagnoses had been given (to these two subjects), including a mood disorder, psychotic disorder NOS, and impulse control disorder. Both subjects were additionally diagnosed with PDD. Despite the identification of catatonia, only one of the two subjects was treated with benzodiazepines. Neither subject was treated with ECT (Electroconvulsive Therapy).

The 18 subjects identified with catatonia by the investigators differed significantly from the rest of the group in being older at the time of data collection $(15.2 \pm 1.5$ vs. $13.7 \pm 2$; df $=99, P=$ $0.003)$ and had a lower score of global functioning $(\mathrm{GAF}=38$ vs. $48 ; \mathrm{df}=84, P=0.04$; the last recorded GAF score). Marked diagnostic differences (diagnoses were made at any time during treatment) included a lower rate for bipolar disorder diagnosis: 1 (5.6) vs. 27 (32.5); lower rate for disruptive behavior disorder: 3 (16.7) vs. 44 (53). However, mental retardation was more common among this group: 9 (50) vs. 12 (14.5). Other

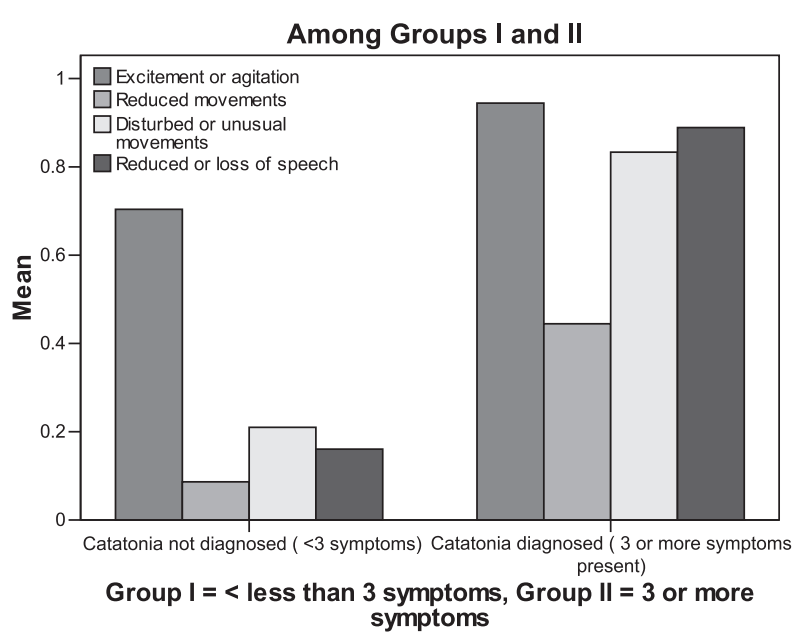

Fig. 1. Symptom-frequency suggestive for catatonia among groups I and II.

significant differences were a greater likelihood of receiving antipsychotics during the past 5 years $(18 / 18$ vs. $45 / 83 ; P=0.00)$, greater likelihood of receiving a benzodiazepine (10/18 vs. 19/83; $P=0.005$ ) (treatment given at any time during service), and a higher likelihood of positive seizure activity was identified on EEG (Electro-Encephalogram) $(2 / 18$ vs. $3 / 83 ; P=0.002)$.

Aggression was more common among the group diagnosed with catatonia compared with the noncatatonic group $\left(\chi^{2}=9.8, \mathrm{df}=2, P=0.008\right)$. Similarly, reduced movements $\left(\chi^{2}=14.7, \mathrm{df}=1\right.$, $P=0.00)$ and loss of speech $\left(\chi^{2}=38.3\right.$, df $=1$, $P=0.00)$ were also more common among this group. Despite the greater frequency of aggression and agitation/excitement, the severity of both symptoms did not differentiate the groups.

Figure 1 describes the frequency of symptoms among groups with and without catatonia. Table 1 describes diagnoses, laboratory findings, symptoms, and treatment-related issues among the entire group and between-group differences (those who met or did not meet criteria).

\section{Discussion}

In summary, our findings are: i) We identified a high rate of catatonia $(17.8 \%)$ using a relatively stringent criterion (presence of three or more symptoms) among a group of children and adolescent clustered by their diagnoses (PDD, mental retardation, psychosis-NOS, and intermittent explosive disorders, catatonia, NMS); ii) Although 'catatonia' was used as a key search term, only two cases had been diagnosed with catatonia by their treatment providers; clearly, the diagnosis is rarely given or treated using established treatments; iii) 


\section{Ghaziuddin et al.}

Table 1. Comparison of diagnoses, laboratory findings, and treatment between subjects diagnosed with and without catatonia

\begin{tabular}{|c|c|c|c|c|}
\hline Variable & $\begin{array}{c}\text { All participants, } \\
n=101\end{array}$ & $\begin{array}{c}\text { Group I (<3 } \\
\text { symptoms), } \\
n=83(82 \%)\end{array}$ & $\begin{array}{c}\text { Group } 2 \text { (3 or } \\
4 \text { symptoms), } \\
n=18(17.8 \%)\end{array}$ & $\begin{array}{c}P \text {-value, (group } \\
\text { I and } 2 \\
\text { comparison) }\end{array}$ \\
\hline Any depression present & $14(13.9)$ & $12(14.5)$ & $2(11)$ & ns \\
\hline Any bipolar disorder present & $28(27.7)$ & $27(32.5)$ & $1(5.6)$ & $0.02^{*}$ \\
\hline Any anxiety disorder present & $19(18.8)$ & $15(18.1)$ & $4(22.2)$ & ns \\
\hline Any disruptive behavior disorder present & $47(46.5)$ & $44(53)$ & $3(16.7)$ & $0.005^{*}$ \\
\hline Mental retardation & $21(20.8)$ & $12(14.5)$ & $9(50)$ & 0.002 \\
\hline Aggression & $71(70.3)$ & $55(66.3)$ & $16(88.9)$ & $0.02^{*}$ \\
\hline Benzodiazepine received & $29(28.7)$ & $19 / 83(22.9)$ & $10 / 18(55.6)$ & $0.005^{*}$ \\
\hline Antipsychotics received during past 5 years & $63(62.4)$ & $45 / 83(55)$ & $18 / 18(100)$ & $0.00^{*}$ \\
\hline ECT received & $2(2.0)$ & $1 / 83(1.2)$ & $1 / 18(5.6)$ & ns \\
\hline Creatinine phosphokinase enzyme measured & $5(5)$ & $3 / 83(3.6)$ & 2/18 (11.1) & ns \\
\hline EEG completed & $15(14.9)$ & $7 / 83(8.4)$ & $8 / 18(44.4)$ & $0.00^{*}$ \\
\hline
\end{tabular}

*Variable differentiates between those with three or more symptoms suggestive of catatonia vs. those with $<3$ symptoms.

Aggression was a common symptom among subjects who met the study-defined criteria for catatonia; however, the severity of aggression did not differentiate the groups; iv) Data pertaining to repetitive or stereotyped movements were rarely documented by treatment providers; v) Psychomotor retardation was less prominent than agitation/excitement among subjects identified with catatonia, suggesting that despite the presence of both retardation and agitation/excitement, the latter symptoms may be the hallmark symptom; vi) Previously known associations, such as male preponderance and association with antipsychotics, were supported by our findings; vii) Only one subject among the entire group of 101 subjects was diagnosed with schizophrenia; this subject did not meet the study-defined criteria for catatonia, thereby lending further support that catatonia is possibly more common among diagnoses other than schizophrenia.

Clinical implications of this study are that catatonia is frequently missed, suggesting that many psychiatrists are possibly unfamiliar with this condition that benzodiazepines are infrequently prescribed and CPK levels and EEG recording are rarely completed. The subgroup with catatonia was found to have poor overall functioning, indicated by a low score of global functioning (last recorded score). Lower symptom-severity scores involving loss of speech/reduced speech and reduced movements, while a higher frequency of aggression and agitation/excitement, suggest that an agitated form of the disorder, rather than a classical 'retarded' form of catatonia, may be more common among this patient group. Further, based on the symptom presentation of this group, this population undoubtedly places enormous burden of care on care-givers and providers alike.
The main strength of this study is that we used stringently defined criteria for catatonia (the presence of three or more symptoms), while DSM-IV requires the presence of only two symptoms for identifying catatonia. Therefore, it is possible that a higher prevalence than $17.8 \%$ may be found if DSM-IV criteria were applied.

Study limitations are retrospective design and variable quality of data obtained by computerassisted chart review. For example, it was not possible to conclude whether the symptoms identified were simultaneously present, determine symptom duration or the exact timing when an antipsychotic or a benzodiazepine was administered.

Our findings suggest that catatonia is not a rare condition among child and adolescent psychiatric patients. This observation is similar to what is noted among adult patients that the condition is underdiagnosed and undertreated. During the early 1980s, catatonia was thought to be almost extinct. However, recent studies find that prevalence rates may range from $7 \%$ to $17 \%$ among adult psychiatric patients with affective or psychotic disorders, who are hospitalized to acute facilities $(13,28)$, suggesting that catatonia is underdiagnosed $(29,30)$. Under diagnosis of catatonia may be due to the historical decision to classify catatonia as a type of schizophrenia, the segregation in long-term facilities of severely ill psychiatric patients and patients with developmental disorders, the perceived lack of anti-catatonic treatments, and the disparagement of physical and neurological examination by psychiatrists (28).

Our study shows that there is a failure to recognize catatonia or treat with effective treatments (lorazepam and ECT) children and adolescents who are receiving psychiatric care. These findings, among other published data, call for an 
urgent need to identify symptoms of catatonia during psychiatric history-taking and examination that psychiatrist must acquire the skills necessary to elicit physical symptoms of catatonia and become familiar with standardized rating scales that are important in diagnosis and measuring symptom severity. Commonly used ratings scales are the Bush Francis Catatonia Rating Scale, Braunig Catatonia Scale, and Northoff Catatonia Rating Scale, which are helpful in identifying and for measuring change in symptoms such as rigidity, grasp reflex, mitgehen, gegenhalten, and reduced inhibitory control $(31,32)$.

Other broader remedial actions may include separating catatonia from psychosis and schizophrenia in psychiatric classification, thereby improving early diagnosis and treatment of catatonia in children and adolescents, especially those with autistic or other developmental disorders (4). The occurrence of catatonia in adolescents with a non-psychotic disorder like autism is an important finding that further loosens the historical link between catatonia and psychosis/schizophrenia. Frequent presence of aggression among subjects diagnosed with catatonia also calls attention to considering catatonia in the differential diagnoses among patients with aggression.

ECT is likely to remain an essential component in the treatment of catatonia. Wider recognition of the benefits of ECT in pediatric patients with catatonia will help reduce stigma of ECT when recommended for children and adolescents or patients with developmental disorders. To a lesser extent, this would also apply to the use of benzodiazepines, which are frowned upon when prescribed in high doses, but often critical for successful treatment. Treatment trials of catatonia are warranted in children and adolescents with affective, psychotic, autistic, developmental, and medical conditions.

\section{Declaration of interest}

Each author acknowledges no financial relationships with commercial interest, which are relevant to this manuscript.

\section{References}

1. Ghaziuddin N, Gih D, Barbosa V, Maixner DF, Ghaziuddin M. Catatonia with onset in puberty. ECT response in 2 adolescents. J ECT 2010;26:270-276.

2. Fink M, TAYlor MA. The many varieties of catatonia. Eur Arch Psychiatry Clin Neurosci 2001;251(Suppl. 1):I8-I13.

3. Francis A, Fink M, Appiani $F$ et al. Catatonia in DSM5. J ECT 2010;26:246-247.

4. Dhossche D, Cohen D, Ghaziuddin N, Wilson C, Wachtel L. The study of pediatric catatonia supports a home of its own in DSM-V. Med Hypotheses 2010;75:558-560.
5. Kahlbaum K. Die Katatonie oder das Spannungsirrensen. Berlin: Verlag, Hirschwald, 1874.

6. Kraeplin E. Psychiatrie: Ein Lehrboch fur Studierende und Aerzte. Aufl Leipzig 1896;5:789-814.

7. Morrison J. Catatonia. Retarded and excited types. Arch Gen Psychiatry 1973;28:39-41.

8. Abrams R. Catatonia. A prospective clinical study. Arch Gen Psychiatry 1976;33:579-581.

9. Taylor MA, Abrams R. Catatonia. Prevalence and importance in the manic phase of manic-depressive illness. Arch Gen Psychiatry 1977;34:1223-1225.

10. Gelenberg A. The Catatonia syndrome. Lancet 1976;1: 1339-1341.

11. Detweiler MB, Mehra A, Rowell T, Kim KY, Bader G. Delirious mania and malignant catatonia. A report of 3 cases and review. [Review] [63 refs]. Psychiatr Q 2009;80:23-40.

12. Val aC, Souza A, Nicolato R, Teixeira Al, Salgado JV. [Catatonic syndrome associated with mixed bipolar disorder: a case report and therapeutic considerations]. [Portuguese]. Revista Brasileira de Psiquiatria 2008;30:399-400.

13. Chalasani P, Healy D, Morriss R. Presentation and frequency of catatonia in new admissions to two acute psychiatric admission units in India and Wales. Psychol Med 2005;35:1667.

14. Wing L, Shah A. A systematic examination of catatonialike clinical pictures in autism spectrum disorders. Int Rev Neurobiol 2006;72:21-39.

15. Rosebush PI, Mazurek MF. Catatonia and its treatment. Schizophr Bull [Comment Review] 2010;36:239-242.

16. Alao AO, Chlebowski S, Chung C. Neuropsychiatric systemic lupus erythematosus presenting as bipolar I disorder with catatonic features. Psychosomatics 2009;50: 543-547.

17. Peralta V, Campos MS, De Jalon EG, Cuesta MJ. DSM-IV catatonia signs and criteria in first-episode, drug-naive, psychotic patients: psychometric validity and response to antipsychotic medication. Schizophr Res 2010;118:168175.

18. Fink M, Shorter E, Taylor MA. Catatonia is not schizophrenia: Kraepelin's error and the need to recognize catatonia as an independent syndrome in medical nomenclature. [Review] [80 refs]. Schizoph Bull 2010;36: 314-320.

19. Ali S, Welch CA, Park LT et al. Encephalitis and catatonia treated with ECT. Cogn Behav Neurol 2008;21:46-51.

20. Ito F, Kondo N, Fukushima S, Suzuki K, Awata S, Matsuoka H. Catatonia induced by idiopathic hypertrophic pachymeningitis. Gen Hosp Psychiatry 2010;32(4):447.e7447.e10.

21. Tueth MJ. Emergencies caused by side effects of psychiatric medications. [Review] [31 refs]. Am J Emerg Med 1994;12:212-216.

22. Baruk H. The battle of schizophrenia. [French]. Bull de 1 Acad Nat Med 1990;174:191-196.

23. Pfister HW, Preac-Mursic V, Wilske B et al. Catatonic syndrome in acute severe encephalitis due to Borrelia burgdorferi infection. Neurology 1993;43:433-435.

24. Snyder S, Prenzlauer S, Maruyama N, Rose DN. Catatonia in a patient with AIDS-related dementia. J Clin Psychiatry 1992;53:414.

25. Wing L, ShaH A. Catatonia in autistic spectrum disorders.[see comment]. Br J Psychiatry 2000;176:357-362.

26. Dhossche DM. Autism as early expression of catatonia. Med Sci Monit 2004;10:RA31-RA39.

27. Seyfried L, Hanauer D, Nease D, Albeiruti R, Kavanagh J, Kales HC. Enhanced identification of eligibility for 


\section{Ghaziuddin et al.}

depression research using an electronic medical record search engine. Int J Med Inform 2009;78:e13-e18.

28. Fink M, TAYLor MA. CATATONIA: a clinician's guide to diagnosis and treatment. Cambridge, UK; New York: Cambridge University Press, 2006.

29. Ungvari GL, Leung S, Ng FS, Cheung HK, Leung T. Schizophrenia with prominent catatonic features ("catatonic schizophrenia"). I. Demographic and clinical correlates in the chronic phase. Prog Neuropsychpharmacol Biol Psychiatry 2005;29:27-38.
30. Van Der Hft S, Arts N, Hoogendoorn M, Kahn R, Verhoeven W. Catatonia disappeared or under diagnosed? Psychopathology 2005;15:3-8.

31. Bush G, Fink M, Petrides G, Dowling F, Francis A. Catatonia. I. Rating scale and standardized examination. Acta Psychiatr Scand 1996;93:129-136.

32. Rooseleer J, Willaert A, Sienaert P. Rating scales for assessing catatonia; which ones are the best? Tijdschrift voor Psychiatrie. [English Abstract Review] 2011;53:287298. 